\title{
Feasibility analysis of yoga on sports fatigue recovery of Competitive Aerobics
}

\author{
Yu Tan, ChengQing Tan \\ College of physical education, Hunan Normal University, Changsha, Hunan 410012 ,China.
}

\begin{abstract}
: competitive aerobics is a group of difficult and beautiful sports with high intensity, high difficulty and remarkable competitiveness. With the strengthening of sports training and competition, sports fatigue occurs from time to time. Some researchers have proved that yoga has a positive effect on the recovery of exercise-induced fatigue through implementation, Through the method of literature, this paper finds that yoga practice is of great significance to the recovery training of sports fatigue, so it puts forward that yoga practice can be applied to the recovery training of sports fatigue in competitive aerobics.
\end{abstract}

Keywords: Yoga; Competitive aerobics; Fatigue recovery

Yoga is a soft, low-intensity exercise, which has a positive effect on the physiological and psychological functions of the human body. The intensity of competitive aerobics is high and the technical difficulty is high. With the development of events, the number of events increases, the training and competition tasks become heavier, and the athletes' sports fatigue is more significant. which are the research on the causes, diagnosis methods and recovery means of sports fatigue of competitive aerobics, and lack of in-depth research on specific methods of fatigue recovery. It is unprecedented to apply yoga practice to the sports fatigue recovery training of competitive aerobics, so this paper puts forward the application of yoga to the sports fatigue recovery of competitive aerobics, and explores its feasibility and practical significance.

\section{1 .related concepts}

\subsection{Competitive Aerobics}

According to the International Gymnastics Federation aerobics competition rules, competitive aerobics is defined as a complete set of movements with continuous, complex and high-intensity characteristics under the accompaniment of music. It is a kind of sports with human health, strength, beauty, difficulty and overall quality ${ }^{[1]}$ 。

\subsection{Yoga}

The Sanskrit of yoga is yuj, which means connection and combination. Yoga,By following the order of body perception, people's body, mind and spirit can develop harmoniously, which is a health care method for people to practice and exercise ${ }^{[2]}$. Yoga is a philosophy, which can recognize oneself by exercising one's body, spirit and consciousness.

Copyright $(\mathcal{C} 2020$ Yu Tan et al.

doi: $10.18282 / 1-$-e.v9i4.1658

This is an open-access article distributed under the terms of the Creative Commons Attribution Non-Commercial License (http://creativecommons.org/licenses/by-nc/4.0/), which permits unrestricted non-commercial use, distribution, and reproduction in any medium, provided the original work is properly cited.

[8] Liu Shuan, Jiang Xin, Zhao Hai-chao, et al. Corrosion Resistance and Wear Property of Graphene-Epoxy Coatings [J]. Tribology, 2015, 35(5): 598-605.

[9] Hu Haixia, Yu Sirong, Ma Jun, Wang Yuhui. Surface chemical effects epoxy dry friction process [J] Tribology, 2007, 27 (3): 241-245.

[10] Wang Yong-kai. Research on preparation and properties of graphene/epoxy resin composites [D]. Zhengzhou: Zhengzhou University, 2013.

[11] CHEN Jian-jian. The interfacial propertie's study of epoxy resin/carbon fiber composite grafted graphene [D]. Wuxi: Jiangnan University, 2012.

[12] Christina A, Hacker, Kelly A, Anderson, Lee J. Comparison of Si-O-C interfacial bonding of alcohols and aldehydes on $\mathrm{Si}(111)$ formed from dilute solution with ultraviolet irradiation[J]. Langmuir 2005, 21: 882-889.

[13] WANG Xin. Functionalization of graphene and its epoxy composites, and study on their flame retardant properties and mechanism [D]. Beijing: University of Science and Technology of China, 2013.

[14] Tanaka K. Friction and wear of semicrystalline polymers sliding against steel under water lubrication [J]. Journal of Lubrication Technology, Transactions ASME, 1980, 102(4): 526-533.

[15] Shi G, Zhang M Q, Rong M Z, et al. Friction and wear of low nanometer Si3N4 filled epoxy composites [J]. Wear, 2003, 254 : 784-796. 


\section{3 exercise fatigue}

Exercise fatigue refers to the phenomenon that the physiological process of the body can not be sustained, and its function can not be maintained at a specific level or predetermined exercise intensity, resulting in the decline of the body's exercise ability ${ }^{[3]}$.

\section{Research on sports fatigue of Competitive Aerobics}

\section{1 characteristics of Competitive Aerobics}

Competitive aerobics is a sub maximum intensity anaerobic exercise project. The change of the new rules requires athletes to pay attention to difficulty, skill, quality and rhythm in a short time, and have outstanding artistic expression and on-site effect ${ }^{[4]}$.

\section{2 causes of fatigue}

Competitive aerobics is mainly based on anaerobic metabolism. According to the research, the main causes of sports fatigue in competitive aerobics are energy and material depletion, internal environment damage, central nervous system imbalance, accumulation of metabolites and increase of free genes ${ }^{[5]}$.

\section{3 performance, judgment of fatigue}

According to different sports fatigue phenomenon, the sports fatigue of competitive aerobics can be divided into three levels, namely mild, moderate and severe fatigue .The commonly used fatigue judgment methods include teaching observation, self observation and physiological diagnosis ${ }^{[5]}$.

\section{Research on yoga in the recovery of exercise-induced fatigue}

\section{1 positive effects of yoga on physical and mental functions}

Yoga has the characteristics of softness, safety and low intensity, and does not involve competitive. Yoga postures emphasize the combination of body and mind, and pay attention to the feeling of the moment ${ }^{[6]}$. The origin of yoga postures is the imitation of various animal and plant postures. Pansare ${ }^{[7]}$ and other studies found that yoga postures can effectively enhance muscle strength, improve stiffness, massage abdominal organs, improve physical quality, and regulate cardiovascular function 。 Yoga rest practice can reduce the excitability of the sympathetic nervous system, and long-term practice can also effectively reduce psychological stress factors ${ }^{[8]}$.

Generally speaking, the influence of yoga practice on physical and psychological functions is closely related to each other. From the physiological level, the positive effect of yoga practice is mainly manifested in the regulation and recovery of human nerve, endocrine, cardiovascular and cerebrovascular system, skeletal muscle system, etc. from the psychological level, it is mainly manifested in the regulation and recovery of consciousness, emotion, mood, self-confidence and psychological state The adjustment of the system.

\section{2 research status of yoga in sports fatigue recovery}

Wang Lei ${ }^{[9]}$ said in the research that meditation practice can make middle and long distance runners well control their own psychology and emotions, and can give full play to their own level in the competition. Ding Zhiyuan ${ }^{[10]}$ found in the study that the method of regulating breath can enhance the lung respiratory function of football players, reduce respiratory fatigue, and have a positive impact on endocrine and digestive organs. Yoga posture practice can help athletes recover the normal $\mathrm{pH}$ value of muscle system and improve sports ability. Gao Jie Mana ${ }^{[1]}$ found in the experimental study that Yoga relaxation can effectively relieve the fatigue of young speed skaters after high-intensity training. Zhang Hui ${ }^{[12]}$ found in the experimental research of basketball players that Yoga can more effectively relieve the negative emotions and bad state of the players due to high-intensity training and competition, effectively adjust the physiological and psychological state of the players, and then improve the performance of the game.

\section{The feasibility analysis of yoga on sports fatigue recovery of Competitive Aerobics}

Many experiments have proved that yoga has a significant recovery effect on exercise-induced physical fatigue and psychological fatigue. it will play an important role to apply yoga to sports fatigue recovery of competitive aerobics. From the recovery of physiological fatigue, scientific yoga practice can alleviate the athlete's heart rate, adjust and restore the nervous system, endocrine system, enhance cardiopulmonary function, reduce the body's lactic acid level, enhance physical fitness, relax muscles, quickly recover heart rate and body energy, improve central nervous fatigue, protect cerebral cortex, improve sleep quality, and enhance the enthusiasm of rest So as to improve the sports ability. From the perspective of psychological fatigue recovery, yoga practice can reduce athletes' subjective fatigue, promote the release of psychological pressure, improve mood, alleviate and avoid bad mood and mental state, and protect athletes' mental health.

\section{5 conclusions and suggestions}

The intensity of competitive aerobics is high and the technical difficulty is high. If the fatigue is not eliminated in time, it will reduce the athletes' sports ability and affect the competition results. Yoga has a positive effect on human body and mind, which can not be replaced by other sports. It has a positive and practical value for the recovery of athletic fatigue of Competitive Aerobics athletes. Coaches should correctly understand the role of yoga in the recovery of fatigue, and apply it to the actual training process, select professional coaches according to the characteristics of competitive aerobics, systematic, scientific, long-term guidance for athletes, so that athletes can correctly understand and master the methods of yoga practice, maximize the positive role of yoga, restore function, improve athletic ability Strength and level. 


\title{
Original Research Article Research on the development of aesthetic education in primary and middle schools
}

\author{
Lei Wang* \\ Jiangsu normal university, Xuzhou, Jiangsu 221006, China.
}

\begin{abstract}
In recent years, social and economic development has been tremendous, but the people's spiritual quality, especially the development of aesthetic ability has been very poor. The exploration of aesthetic education has become an important node of liberal education and Education. For All, for the law and characteristics of aesthetic education in education and the development of campus aesthetic education put forward higher requirements and we need to continue to explore the process of aesthetic education and expand the status of education and aesthetic education. From the angle of aesthetics and pedagogy, following the characteristics of aesthetic education, this paper studies the development of aesthetic education and campus aesthetic education, aiming at improving the teaching of aesthetic education. With a view to improving the personal development of students, as well as the spirit of the whole society.
\end{abstract}

Keywords: Aesthetic Education; Aesthetic Education Teachers; Professional Quality; Campus Aesthetic Education

Aesthetic education leads us to have a pair of eyes that are good at discovering the beauty around us. Aesthetic education has brought up the spirit, culture, morality and other aspects of the Chinese nation, and has continuously sent fresh blood to the nation, it has laid a solid foundation for the realization of the Chinese dream.

Copyright (C) 2020 Lei Wang

doi: 10.18282/1-e.v9i4.1659

This is an open-access article distributed under the terms of the Creative Commons Attribution Non-Commercial License (http://creativecommons.org/licenses/by-nc/4.0/), which permits unrestricted non-commercial use, distribution, and reproduction in any medium, provided the original work is properly cited.

\section{Reference}

[1] Han Mengmeng, Zhu Sai, Wu Xiaohong. Research on sports fatigue and nutritional recovery of Competitive Aerobics athletes [J]. Combat (Martial Arts Science), 2014,11 (02): 114-116.

[2] Gao Chengli. Correct understanding and application of Yoga [J]. Sports science and technology literature bulletin, 2013,21 (03): 94-95.

[3] Deng Jia. Research on sports fatigue and recovery theory in track and field training [J]. Sci tech information, 2011 (10): 220 221.

[4] Liu Jiangbo, Li Jing, Li Yang, Cheng Yiran. Analysis on the development of Competitive Aerobics under the new rules of Competitive Aerobics in fig2017-2020 cycle [J]. Scientific mass (Scientific Education), 2020 (03): 188.

[5] Sports Fatigue Diagnosis of Aerobics Athletes in Liaoning Province, 2004.

[6] Du Xiru, Zhang linting. Experimental study on the influence of yoga on College Students' physical fitness and mental health

(J). Journal of Capital Institute of physical education, 2005 (01): 100-101.

[7] PansareMS, KulkarniAN, PendseUB. EffectofyogictrainingonserumLDHlevels [J] . JournalofSportsMedicine\& Physi calFit-ness, 1989, 29(2):177-178.

[8] The Effectsof Participating Hatha Yogaon the Physical-Psychological Functional Recoveryof Female University Students.2017,56(5):157-171.

[9] Wang Lei, Yi Xingfeng. Application of yoga in recovery training of middle and long distance running [J]. Journal of Social Sciences of Jiamusi University, 2011,29 (05): 168-170.

[10] Ding Zhiyuan. Research on yoga for football sports fatigue recovery [J]. Science and technology prospect, 2016,26 (05): 278.

[11] Gao Jie. Experimental study on the effect of yoga on fatigue recovery of Latin dance [D]. Xi' an Institute of physical education, 2015.

[12]Zhang Hui. Experimental research on yoga in basketball players' fatigue recovery after intensive training [D]. Jilin Institute of physical education, 2017. 